RESEARCH ARTICLE

Cross-cultural adaptation and validation of Sinhalese version of the short-form of Child Perception Questionnaire 11-14 to determine oral health related quality of life of adolescents

B.K.G. Thilakarathne* and L. Ekanayake

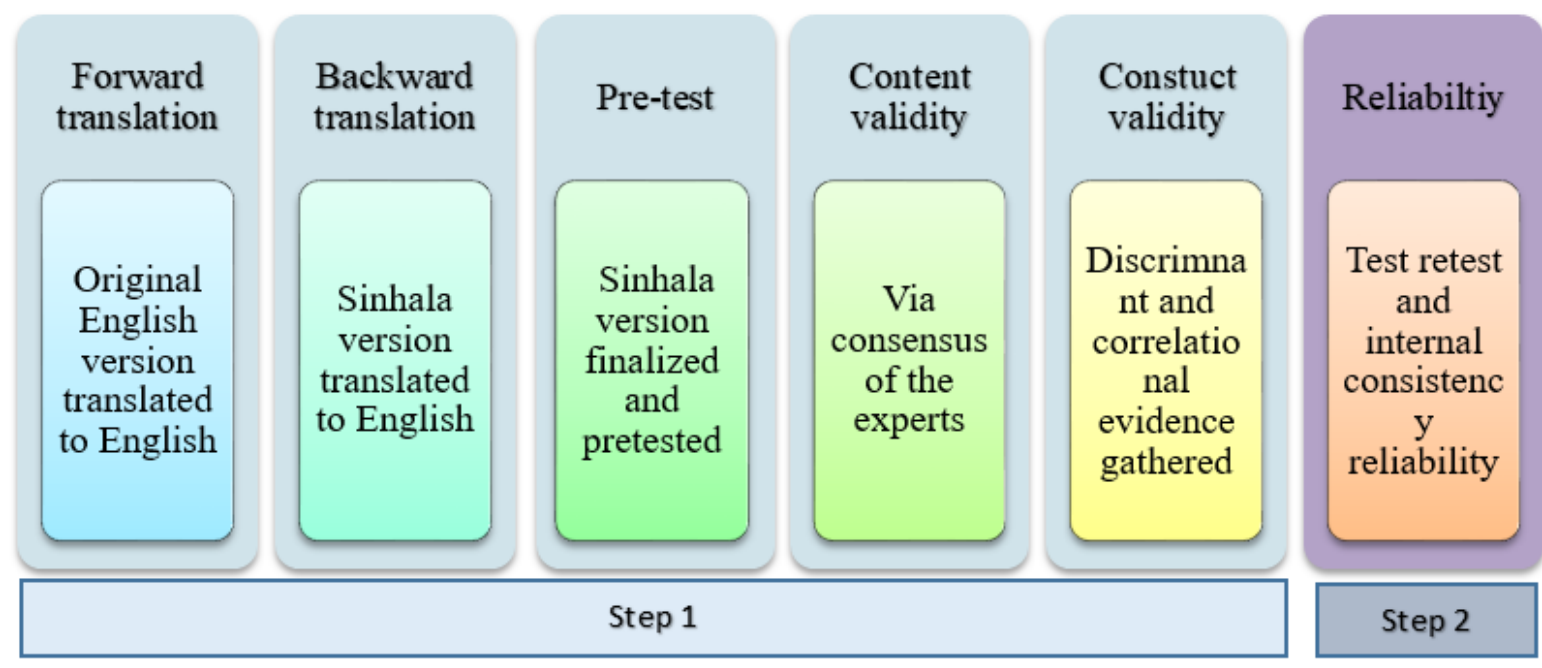

\title{
Highlights
}

- Oral health related quality of life instruments are cross- culturally adapted and validated to suit the populations of interest.

- The Child Perception Questionnaire (CPQ11-14) is an instrument widely used to assess oral health related quality of life.

- CPQ11-14 Sinhalese version showed adequate internal consistency, construct validity based on correlational evidence and test-retest reliability.

- CPQ11-14 is a valid instrument to determine OHQOL in 15-year-old Sinhala speaking children. 


\title{
RESEARCH ARTICLE
}

\section{Cross-cultural adaptation and validation of Sinhalese version of the short-form of Child Perception Questionnaire 11-14 to determine oral health related quality of life of adolescents}

\author{
B.K.G. Thilakarathne* and L. Ekanayake
}

Division of Community Dentistry, Department of Community Dental Health, Faculty of Dental Sciences, University of Peradeniya, Peradeniya, Sri lanka.

\section{Received: 09/11/2020; Accepted: 04/05/2021}

\begin{abstract}
It is now well established that oral health cannot be assessed merely by clinical indicators but also complemented with subjective measures. This study aimed to validate the Sinhalese version of the Child Perception Questionnaire (CPQ11-14). The English version of the CPQ11-14 was translated to Sinhala and face and content validity were assessed. The Sinhala version of the CPQ11-14 was then administered to a sample of 150, fifteenyear-old school children from Kurunegala district. Discriminant validity was determined by comparing total CPQ scores with severity of dental caries, dental fluorosis and malocclusion status. Convergent validity was determined by assessing the correlations between global indicators of oral health and total and subscales of the CPQ. In order to check for test-retest reliability, the questionnaire was administered to the same group of students two weeks' a part and Intra- class Correlation Coefficient (ICC) was assessed. Internal consistency was assessed using Cronbach's alpha. There were significant correlations between the global indicators and total and subscales of CPQ11-14 thus ensuring convergent validity. The severity of dental caries, dental fluorosis or malocclusion was not significantly associated with the total CPQ scores and thus discriminant validity could not be proven. Cronbach's alpha for the total scale was 0.8 and ranged from 0.4-0.7 for subscales. ICC for the total scale was 0.9 and ranged from 0.7 to 0.8 for the different subscales. The Sinhala translation of the short-form CPQ11-14 questionnaire showed adequate internal consistency, convergent validity and test-retest reliability. Therefore, it is a valid instrument to determine OHQOL in 15-year-old Sinhala speaking children.
\end{abstract}

Keywords: Child Perception Questionnaire; oral health related quality of life; Sinhalese; cross- cultural adaptation.

\section{INTRODUCTION}

Health status has been mainly measured using clinical indicators which is based on the "biomedical" paradigm of health. However in addition to the presence or absence of disease, it is now well established that any measure of health should assess psycho-social aspects of health as well (Allen, 2003). Health related quality of life and oral health related quality of life (OHQOL) instruments have been developed considering this point of view (Sheiham and Croog, 1981).

OHQOL instruments have been used for different purposes such as political, clinical, research and public health (Robinson, 2016). There are three measures of OHQOL and they include social indicators, global selfratings of $\mathrm{OHQOL}$ and multiple-item questionnaires (Slade, 2002). Multiple item questionnaires are classified into generic and disease specific types and widely used to assess OHQOL (Sischo and Broder, 2011).

In children, OHQOL is assessed using various instruments. A recent systematic review on oral health related quality of life instruments available for children and adolescents has reported that the Child Perceptions Questionnaire 11-14 (CPQ11-14) is the best rated instrument to assess OHQOL in school children and adolescents (Zaror et al., 2019). The CPQ11-14 was developed by Jokovic et al in 2002 (Jokovic, et al., 2002) and includes 36 items. This self-administered questionnaire is subdivided into four subscales; oral symptoms, functional limitations, emotional well-being and social well-being. As the original CPQ included 36 items and therefore timeconsuming to administer, several shorter versions such as the 8- and 16- item CPQ have been developed and shown to be valid and reliable in assessing OHQOL in adolescents (Jokovic, et al., 2006). Item impact method was used to develop ISF versions (16 and 8) and regression method was used to develop RSF versions (16 and 8). The short version of CPQ11-14 ISF 16 has been translated into different languages and validated in different populations (Gururatana, et al., 2011; Mohamed, et al., 2013; Torres, et al., 2009).

There is a paucity of information related to OHRQOL in Sri Lankan adolescents. In order to assess OHRQOL in Sri Lankan adolescents it is necessary either to develop a new instrument or translate and validate an existing instrument that has been developed specifically to examine OHRQOL in this group. Validating an existing instrument is a better option as it would be less costly and time consuming. The CPQ11-14 was developed after considering many conditions that affect children's daily lives and also it is the most widely used in research. Further it has high evidence for its reliability and validity (Gilchrist, et al., 2014). Therefore the aim of this study was to assess the psychometric properties of the Sinhala version of the CPQ11-14 ISF:16 so that it could be used to assess oral health related quality of life of Sri Lankan adolescents in future research. 


\section{MATERIALS AND METHODS}

This validation study is the first component of an extensive study that was conducted to assess dental fluorosis among 15 -year-old school children in Kurunegala district. Ethical clearance for the study was taken from Ethics Review Committee, Faculty of Medicine, University of Colombo (EC-17-016). Permission was also obtained from the Provincial Director of Education of the North Western Province, the relevant zonal education directors and school principals. The Short form of the Child Perception Questionnaire includes a total of 16 items in four subscales; four items on oral symptoms, four items on functional limitations, four items on emotional wellbeing and four items on social wellbeing. The responses to each item were recorded on a 5-point Likert scale; never $=1$, once or twice $=2$, sometimes $=3$, often $=4$ and every day or almost every day $=5$. The total score therefore ranges from 16 to 80. A higher CPQ total score indicates poorer oral health related quality of life. Subscale scores are also calculated by summing up scores for items in each subscale.

The methods recommended by Beaton, et al., (1998) were used for translation and cross-cultural adaptation of the CPQ11-14. The original English version of the CPQ11-14 ISF:16 questionnaire was translated to Sinhala by two persons; the principal investigator (PI) with the knowledge about oral health related quality of life and a teacher of English. A group consisting of the PI, teacher of English and Professor of Community Dentistry checked the two translated versions for agreement and a draft of the Sinhala version was developed based on the two Sinhala translations which were then back translated to English by two sworn translators to ensure accuracy and comparability of the translation. A panel comprising of an expert in Dental Public Health, translators, and health professionals compared, the draft Sinhala version, the two English translations of the Sinhala draft and the original CPQ for cultural and item equivalence. Based on their comments the second draft of the Sinhala version was developed. This draft Sinhala version was then pretested among a convenient sample of 30 students (15 girls and 15 boys) attending Mawathagama Central College, Kurunegala. Based on the feedback received from the students, minor modifications in language were made to improve clarity of the questions at this stage and the final version of Sinhala CPQ11-14 was developed.

Internal validity of the Sinhala translation of CPQ was assessed through content and construct validity.

Content validity measures the extent to which the items of the questionnaire adequately cover the domain under investigation, and achieved by balanced analysis of the instrument by experts in the field (Price, et al., 2015). A professor in Community Dentistry and a consultant in Dental Public Health checked the items in the questionnaire for relevance, clarity and comprehensiveness thus ensuring content validity. Based on their suggestions certain items were modified.

Construct validity assesses the degree to which the questionnaire measures the construct (oral health related quality of life) (Price, et al., 2015) and was assessed through discriminant and convergent validity. Discriminant validity was determined by comparing total CPQ scores with severity of dental caries, dental fluorosis and malocclusion status. The hypothesis used in assessing discriminant construct validity, was that severe forms of the above conditions will have high CPQ scores compared to lower scores for milder forms. Convergent validity was determined by assessing the correlations between CPQ scores and the global rating of oral health; "What do you think about the condition of your teeth" rated on a 5-point scale ranging from 1 to 5; excellent, very good, good, fair and poor.

The study population consisted of Grade 10 students who had reached their $15^{\text {th }}$ birthday but not the $16^{\text {th }}$ attending public schools in Kurunegala district. This age group was selected to investigate the full effects of fluorosis on the permanent dentition. Those who were permanent residents of the area were included, while those with learning difficulties, wearing fixed orthodontic appliances and who were absent on the day of the examination were excluded from the study. As this study was the first part of a large study on dental fluorosis, the sample size was considered as 150 by according to the similar validation studies (Jokovic, et al., 2006; Gururatana, et al., 2011).

Among the 30 divisional secretary divisions (DS) in Kurunegala district, five DS divisions where dental fluorosis is endemic and another five where dental fluorosis is not endemic were selected randomly ensuring the inclusion of participants with dental fluorosis. From the ten selected DS areas, one school from each DS division was selected randomly. From the ten selected schools, a convenient sample of fifteen students from each school was selected.

Dental fluorosis was assessed using the Thylstrup and Fejerskov Index (TF Index) (Thylstrup and Fejerskov, 1978). Dental caries was assessed using the WHO Basic Methods (WHO, 2013). Dental caries was summarized to Decayed Missed and Filled Surface (DMFS) scores. Dental Aesthetics Index (DAI) was used to assess malocclusion status (Cons, et al., 1986). Prior to conducting the study, the PI was calibrated against specialists in Restorative Dentistry and Orthodontics on the use of relevant clinical indices. A pre-intern medical officer trained by the PI, recorded the data.

The PI randomly selected 15 students from the list and the principals were requested to hand over the information sheets, consent and assent forms to those 15 students which were sent prior to the visit.

On the day of the visit, the PI selected a place with adequate day light to conduct the oral examination. The CPQ was administered to those students who had given their consent and assent. Following the administration of the questionnaire, the PI conducted the oral examination in natural daylight while the participant was seated on a mobile dental chair in the same room where the questionnaires were administered. The assistant recorded the data.

Prior to recording dental fluorosis status, teeth were 
wiped with a piece of sterile gauze. A sterile mirror and a Community Periodontal Index (CPI) probe was used to detect dental caries while a sterile mirror and steel ruler cleaned with surgical spirit were used to record the DAI.

Statistical Package for Social Sciences for windows version 20 (SPSS) and Microsoft Excel were used in data analysis. Reliability of the questionnaire was assessed using internal consistency and test-retest reliability. Testretest reliability of the questionnaire was checked by administrating the CPQ11-14 questionnaire two weeks apart to the first eight of the 15 students in the list from each school. On the second occasion, the questionnaires were posted with a self-addressed stamped envelope to the selected eight students and they were requested to send them to the PI after completion.

As the distribution of CPQ scores was skewed, nonparametric tests were used in the analysis. Krushkal-Wallis test was used to compare the differences in CPQ total scores between groups. Spearmen's correlation coefficient was used to determine the correlation between global rating of oral health and total and subscale scores of CPQ. Internal consistency reliability was assessed by Cronbach's alpha.
Test-retest reliability was assessed by means of intraclass correlation coefficients (ICC) calculated by one-way random-effects model.

\section{RESULTS AND DISCUSSION}

One hundred and fifty students participated in this validation study. Nearly $53 \%$ of the sample consisted of girls.

When the CPQ questionnaires were checked for completeness, it was evident that the participants had responded to all items. Two students (1.3\%) had obtained the minimum score of 16 (floor effect). None of the students had obtained the maximum score of 80 (ceiling effect). The highest score recorded was 52 . The mean total CPQ score was $27.7(\mathrm{SD}=7.5)$ and the median was 25.5 with a range of $16-52$.

Convergent validity was determined by assessing the correlations between the global rating of oral health and total and subscale scores of Child Perception Questionnaire (CPQ). The total score as well as subscale scores of CPQ positively and significantly correlated with the global rating of oral health; perceived condition of teeth (Table 1).

Table 1: Correlation between the perceived condition of teeth and the total and subscale in CPQ scores

\begin{tabular}{lcc}
\hline CPQ scores & Perceived condition of teeth & *p value \\
\hline Total CPQ score & 0.4 & $\mathrm{p}<0.001$ \\
Oral symptoms domain & 0.2 & 0.012 \\
$\begin{array}{l}\text { Functional limitations } \\
\text { domain }\end{array}$ & 0.2 & 0.019 \\
$\begin{array}{l}\text { Emotional well-being } \\
\text { domain }\end{array}$ & 0.4 & $\mathrm{p}<0.001$ \\
Social wellbeing domain & 0.4 & $\mathrm{p}<0.001$ \\
\hline *p value based on Spearman's correlation analysis &
\end{tabular}

Table 2: Associations between severity of oral disease and CPQ scores

\begin{tabular}{|c|c|c|}
\hline \multirow{2}{*}{ Severity of disease entity } & \multicolumn{2}{|r|}{ Total CPQ score } \\
\hline & Mean rank & *p value \\
\hline \multicolumn{3}{|l|}{ Dental caries } \\
\hline $\mathrm{DMFS}=0(\mathrm{n}=100)$ & 71.5 & $* \mathrm{KW} \chi 2=4.051, \mathrm{p}=0.132$ \\
\hline $\mathrm{DMFS}=1-2(\mathrm{n}=23)$ & 75.5 & \\
\hline DMFS $\geq 3(n=27)$ & 90.4 & \\
\hline \multicolumn{3}{|l|}{ Dental fluorosis } \\
\hline $\mathrm{TF}=0(\mathrm{n}=10)$ & 83.8 & $* \mathrm{KW} \chi 2=2.02 ; \mathrm{p}=0.568$ \\
\hline $\mathrm{TF}=1(\mathrm{n}=44)$ & 68.3 & \\
\hline $\mathrm{TF}=2-3(\mathrm{n}=82)$ & 77.2 & \\
\hline $\mathrm{TF} \geq 4(\mathrm{n}=14)$ & 82 & \\
\hline \multicolumn{3}{|l|}{ Orthodontic treatments need } \\
\hline DAI score $\leq 25(\mathrm{n}=78)$ & 73.5 & ${ }^{*} \mathrm{KW} \chi^{2}=2.34, \mathrm{p}=0.568$ \\
\hline DAI score $=26-30(n=32)$ & 73.3 & \\
\hline DAI score $=31-35(\mathrm{n}=20)$ & 89.3 & \\
\hline DAI score $\geq 36(n=20)$ & 73 & \\
\hline
\end{tabular}


The results related to discriminant validity are shown in Table 2. The severity of dental caries, dental fluorosis or malocclusions was not significantly associated with the total CPQ scores.

Cronbach's alpha for the total scale was 0.8 and the Cronbach's alpha values ranged from $0.4-0.7$ for the different subscales (Table 3). Test-retest reliability was assessed by administering the CPQ11-14 questionnaire to the same group of students two weeks apart. Although questionnaires were posted to 80 students, only 51 students had returned the questionnaires giving a response rate of $63.75 \%$. When test-retest reliability was assessed using the ICC, it was found to be 0.9 for the total items of questionnaire. This indicates good agreement (Table 3).

According to the results, the mean total CPQ score was 27.7 ( $\mathrm{SD}=7.5)$. No student obtained the maximum score of 80 (ceiling effect) and only 2 students (1.3\%) had obtained the minimum score of 16 (floor effect). Floor or ceiling effect is considered to be present if $15 \%$ or more of the respondents obtain the maximum or minimum score, and therefore affecting content validity and responsiveness of an instrument (Terweea, et al., 2007). In the study where Jokovic et al., (2002) had validated the original 36 item $\mathrm{CPQ}$, the mean total score reported was $26.3(\mathrm{SD}=16.7)$. However, Jokovic had not reported a floor effect or ceiling effect.

Torres et al., (2009) assessed the Brazilian version of the of the CPQ11-14 for its psychometric properties and had reported that the mean CPQ score for a sample of 11-14year olds was $11.9(\mathrm{SD}=7.6)$. This indicates that the overall OHQOL of those children were better than the participants of the present study. A lower CPQ score indicates better OHQOL. However, similar to the present study only a nonsignificant floor effect (3\%) was observed. In a study to validate the Thai version of the CPQ (Gururatana, et al., 2011), the mean CPQ recorded was $13.4(\mathrm{SD}=6.1)$. The difference between the present study and the Thai study may be due to the differences in recording the responses to the items in the CPQ questionnaire. In the Thai study the responses were recorded on a 0-4 scale while in the present study a 1-5 scale was used. Socio-cultural differences in the perception of oral health may also be a reason for variations in CPQ scores in different communities.
When discriminant validity was determined by assessing the associations between CPQ scores and severity of oral diseases/conditions, significant associations were not observed. Similar findings have been reported in studies where the CPQ11-14 had been translated to other languages and validated among 11-14-year old school children (Barbosa, et al.,2009; Mohamed, et al., 2013). However, in the original study, Jokovic et al., (2002) was able to detect a significant difference between different oral diseases and CPQ score. The study by Kumar et al., (2016) using a sample from the general population had compared various severities of dental caries, presence or absence of malocclusions and various severities of dental fluorosis. That study had shown a statistically significant association between CPQ score and malocclusions only. Dental caries and fluorosis were not significantly associated with CPQ scores. A plausible explanation for the difference in Jokovic's study may be due to the fact that the original CPQ was validated among children attending Pediatric dentistry, Orthodontic and Cranio-facial clinics and how much each of these conditions affect quality of life can vary. It is also reasonable to assume that children attending dental clinics are likely to have more severe forms of oral/dental problems which lead to pain and suffering. As a result, severe forms of oral diseases can affect the oral health related quality of life of individuals.

When Cronbach's alpha was used to assess the reliability of the CPQ11-14 questionnaire, it was found to be 0.8 for the total scale and 0.4-0.7 for the different subscales. Internal consistency is considered to be high if Cronbach's alpha value is 0.7 or above (Dean, et al., 2018). In a study conducted among 11-14-year-old Thai children it has been reported that Cronbach's alpha was 0.9 for the total scale (Gururatana, et al., 2011). Similar results have been reported in other studies as well (Mohamed, et al., 2013). But the internal consistency recorded for some subscales in this study were lower than the recommended value of 0.7 and may be due to the limited number of items in each subscale. Similar findings have been reported in other studies as well. In a study where the Brazilian version of CPQ 11-14 ISF:16 was validated Cronbach's alpha values for subscale ranged 0.5-0.7 (Barbosa, et al., 2009). Testretest reliability was measured using intra-class correlation coefficients (ICC). A duration of two weeks was selected between the two administrations to avoid recall bias and

Table 3: Reliability statistics of CPQ11-14

\begin{tabular}{lcccc}
\hline CPQ11-14 & No of items & Cronbach's $\boldsymbol{\alpha}$ & ICC & $\mathbf{9 5 \%}$ CI of ICC \\
\hline $\begin{array}{l}\text { Total score } \\
\text { Subscales }\end{array}$ & 16 & 0.8 & 0.9 & $0.8-0.9$ \\
Oral symptoms & 4 & 0.4 & 0.7 & $0.5-0.8$ \\
$\begin{array}{l}\text { Functional } \\
\text { limitation }\end{array}$ & 4 & 0.5 & 0.8 & $0.7-0.9$ \\
Emotional & 4 & 0.6 & 0.7 & $0.6-0.8$ \\
wellbeing & & & & $0.7-0.8$ \\
Social wellbeing & 4 & 0.7 & 0.8 & \\
\hline $\begin{array}{l}\text { ICC =Intra class correlation coefficient } \\
\text { One-way random effects model where people effects are random. }\end{array}$ &
\end{tabular}


to minimize the effect due to any clinical changes. The ICC value for the total scale was 0.9 and the subscales scores ranged from 0.7 to 0.8 . A value 0.7 or above is recommended satisfactory (Alphin, et al., 2015). In a study where the 36 item CPQ11-14 questionnaire was validated among Telugu speaking Indian Children, the ICC value reported for the total scale was 0.92 (Kumar, et al., 2016).

\section{CONCLUSIONS}

The Sinhala version of the short form CPQ11-14 questionnaire showed adequate internal consistency, convergent validity and test-retest reliability. Therefore, it appears to be a valid instrument to determine OHQOL in 15-year-old Sinhala speaking children.

\section{ACKNOWLEDGEMENTS}

This study was supported by the Research Grant (RG/2016/84/D) received from University of Peradeniya, Sri Lanka.

\section{DECLARATION OF CONFLICT OF INTEREST}

The authors declare that they do not have any conflict of interests.

\section{REFERENCES}

Allen, P.F. (2003). Assessment of oral health related quality of life. Health and Quality of Life Outcomes 1(40): 1-8, DOI: 10.1186/1477-7525-1-40.

Alphin, S.H. (2015). The Mac New Heart Disease HealthRelated Quality of Life questionnaire: A Scandinavian validation study. Social Indicators Research 122(2): 519-537, DOI: 10.1007/s11205-014-0694-7.

Barbosa, T.S., Tureli, M.M. and Gavião, M.D. (2009). Validity and reliability of the Child Perceptions Questionnaires applied in Brazillian children. BMC Oral Health 9(13), DOI: https://doi.org/10.1186/14726831-9-13.

Beaton, D., Bombardier, C., Guillemin, F. and Ferraz, M.B. (1998). Recommendations for the cross-cultural adaptation of health status measures. American Academy of Orthopaedic Surgeons Institute for Work \& Health, DOI: 10.1097/00007632-200012150-00014.

Cons, N.C., Jenny, J. and Kohout, F. (1986). The Dental Aesthetics Index. Iowa: Iowa University Press.

Dean, K., Walker, Z. and Jenkinson, C. (2018). Data quality, floor and ceiling effects, and test-retest reliability of the Mild Cognitive Impairment Questionnaire. Patient Related Outcome Measures 9: 43-47, DOI: https://doi. org/10.2147/PROM.S145676.

Gilchrist, F., Rodd, H., Deery, C. and Marshman, Z. (2014). Assessment of the quality of measures of child oral health-related quality of life. BMC Oral Health 14(40).

Gururatana, O., Baker, S. and Robinson, P.G. (2011). Psychometric properties of long and short forms of the Child Perceptions Questionnaire (CPQ11-14) in a Thai population. Community Dental Health 28: 232-237.

Jokovic, A., Locker, D., Stephens, M., Kenny, D., Tompson, B. and Guyatt, G. (2002). Validity and reliability of a questionnaire for measuring child oral-health-related quality of life. Journal of Dental Research 81(7): 459-
463, DOI: $10.1177 / 154405910208100705$.

Jokovic, A., Locker, D. and Guyatt, G. (2006). Short forms of the Child Perceptions Questionnaire for 11-14-yearold children (CPQ11-14): development and initial evaluation. Health and Quality of Life Outcomes 4(4), DOI: $10.1186 / 1477-7525-4-4$

Kumar, S., Kroon, J., Lalloo, R. and Johnson, N.W. (2016). Psychometric properties of translation of the Child Perception Questionnaire (CPQ11-14) in Telugu speaking Indian children. PLOS ONE 1(3).

Mohamed, A.R., Thomson, W.M. and Foster Page, L.A. (2013): Validation of a Malay version of the shortform Child Perceptions Questionnaire (CPQ11-14) in Brunei. Brunei Darussalam Journal of Health 5: 56-69.

Price, P.C., Jhangiani, R.S. and Chiang, I.C.A. (2015). Reliability and validity of measurement. In: Research methods in psychology, Canada: Independently published; 87-92.

Robinson, P.G. (2016). Choosing a measure of Health related quality of life. Community Dental Health 33(2):107-115.

Sheiham, A. and Croog, S.H. (1981). The psychosocial impact of dental diseases on individuals and communities. Journal of Behavioral Medicine 4(3): 257-272.

Slade, G.D. (2002). In: Inglehart MR, Bagramian RA (eds.) Oral Health-Related Quality of Life. Quintessence Publishing, Illinois, DOI: 10.1177/0022034511399918

Sischo, L. and Broder, H.L. (2011). Oral health-related quality of life, what, why, how, and future implications. Journal of Dental Research 90(11): 1264-1270, DOI: 10.1177/0022034511399918.

Terweea, C.B., Bota, S.D., de Boer, M.R., van der Windt, D.A., Knol, D.L., Dekker, J., Bouter, L.M. and de Vet, H.C. (2007). Quality criteria were proposed for measurement properties of health status questionnaires. Journal of Clinical Epidemiology 60(1): 34-42, DOI: 10.1016/j.jclinepi.2006.03.012.

Thylstrup, A. and Fejerskov, O. (1978). Clinical appearance of dental fluorosis in permanent teeth in relation to histologic changes. Community Dent Oral Epidemiol 6(6): 315-328, DOI: 10.1111/j.1600-0528. 1978. tb01173.x.

Torres, C.S., Paiva, S.M., Ramos-Jorge, M.L., Vale, M.P., Pordeus, I.A., Oliveira, A.C. and Allison, P.J. (2009). Psychometric properties of the Brazilian version of the Child Perceptions Questionnaire (CPQ11-14)short forms. Belo Horizonte, Health Quality of Life Outcomes 7(43), DOI: 10.1186/1477-7525-7-43.

World Health Organization (2013). Oral Health Surveys: Basic methods. $5^{\text {th }}$ edition, Geneva: World Health Organization.

Zaror, C., Pardo, Y., Espinoza-Espinoza, G., Pont, À., Muñoz-Millán, P., Martínez-Zapata, M.J., Vilagut, G., Forero, C.G., Garin, O., Alonso, J. and Ferrer, M. (2019). Assessing oral health-related quality of life in children and adolescents: a systematic review and standardized comparison of available instruments. Clinical Oral Investigations 23: 65-79, DOI: 10.1007/ s00784-018-2406-1. 